\title{
Investigation of Gas Heating by Nanosecond Repetitively Pulsed Glow Discharges Used for Actuation of a Laminar Methane-Air Flame
}

Deanna A. Lacoste, Byeong Jun Lee, Aman Satija, S. Krishna, Scott A. Steinmetz, Issam Alkhesho, Omar Hazzaa, Robert P. Lucht, Min Suk Cha \& William L. Roberts

To cite this article: Deanna A. Lacoste, Byeong Jun Lee, Aman Satija, S. Krishna, Scott A. Steinmetz, Issam Alkhesho, Omar Hazzaa, Robert P. Lucht, Min Suk Cha \& William L. Roberts (2017): Investigation of Gas Heating by Nanosecond Repetitively Pulsed Glow Discharges Used for Actuation of a Laminar Methane-Air Flame, Combustion Science and Technology, DOI: 10.1080/00102202.2017.1333984

To link to this article: http://dx.doi.org/10.1080/00102202.2017.1333984

Accepted author version posted online: 24 May 2017.

Submit your article to this journal $\pi$

Џ Article views: 28

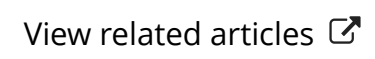

View Crossmark data $\asymp$ 


\section{Investigation of Gas Heating by Nanosecond Repetitively}

\section{Pulsed Glow Discharges Used for Actuation of a Laminar \\ Methane-Air Flame}

Deanna A. Lacoste ${ }^{1}$, Byeong Jun Lee ${ }^{2}$, Aman Satija ${ }^{3}$, Krishna S. ${ }^{1}$, Scott A. Steinmetz ${ }^{1}$, Issam Alkhesho ${ }^{1}$, Omar Hazzaa ${ }^{1}$, Robert P. Lucht ${ }^{3}$, Min Suk Cha ${ }^{1}$, and William L. Roberts ${ }^{1}$

${ }^{1}$ Clean Combustion Research Center, King Abdullah University of Science and Technology, Thuwal, Saudi Arabia

${ }^{2}$ Yeungnam University, School of Mechanical Engineering, Gyeongsan, Korea

${ }^{3}$ School of Mechanical Engineering, Purdue University, West Lafayette, Indiana, USA

This paper reports on the quantification of the heating induced by nanosecond repetitively pulsed (NRP) glow discharges on a lean premixed methane-air flame. The flame, obtained at room temperature and atmospheric pressure, has an Mshape morphology. The equivalence ratio is 0.95 and the thermal power released by the flame is $113 \mathrm{~W}$. The NRP glow discharges are produced by high voltage pulses of $10 \mathrm{~ns}$ duration, $7 \mathrm{kV}$ amplitude, applied at a repetition frequency of $10 \mathrm{kHz}$. The average power of the plasma, determined from current and voltage measurements, is $1 \mathrm{~W}$, i.e. about $0.9 \%$ of the thermal power of the flame. Broadband vibrational coherent anti-Stokes Raman spectroscopy of nitrogen is used to determine the 
temperature of the flame with and without plasma enhancement. The temperature evolution in the flame area shows that the thermal impact of NRP glow discharges is in the uncertainty range of the technique, i.e., +/- $40 \mathrm{~K}$.

Keywords: Atmospheric pressure plasma, Plasma-assisted combustion, Coherent Anti-Stokes Raman Spectroscopy.

\section{INTRODUCTION}

In the last decades, non-equilibrium plasmas produced by nanosecond repetitively pulsed (NRP) discharges have shown promising ability for combustion enhancement (e.g., Starikovskiy et al., 2013; Starikovskaia, 2014). For example, a significant reduction of the ignition delay time (e.g., Yin et al., 2011), and of the lean-flammability limit (e.g., Pilla et al., 2006; 2008), as well as the control of flame dynamics (e.g., Lacoste et al., 2013a; 2013b) can be achieved with the aid of NRP discharges. Their efficiency is attributed to a coupled thermal and chemical activation of the reacting flows (e.g., Do et al., 2010; Tholin et al., 2014; Ju et al., 2015). However, a clear understanding of the physicochemical mechanisms of the NRP discharge action on the aforementioned flame behavior is not yet achieved.

At atmospheric pressure and room temperature, NRP discharges can stabilize in different regimes (Pai et al., 2010). The NRP spark regime is the most energetic regime with a strong gas heating (up to a few thousands of Kelvin) and a significant chemical effect demonstrating up to $50 \%$ of molecular oxygen dissociation (Stancu et al., 2010). This regime is commonly used for combustion enhancement studies because it has the strongest impact. However, due to gas heating, these NRP spark discharges can significantly increase the density of nitric oxide (NO) in the burnt gases (e.g., Lacoste et al., 2013c), which might be a barrier for their use in industrial applications. In order to promote chemical activation, the NRP glow regime (Pai et al., 2009) 
would be preferred. Recently, Lacoste et al. (2017a; 2017b), have shown that NRP discharges in the glow regime, generated in the combustion area, can have a strong impact on the dynamics of laminar premixed flames. The methane-air flame was highly responsive to plasma forcing, in a manner comparable to acoustic modulation of the incoming flow.

Because the energy deposition in the NRP glow regime is very low (a few hundreds of $\mu \mathrm{J}$ per pulse), it is usually admitted that the thermal effect of NRP glow discharges can be neglected. Nonetheless, since accurate thermometry of highly reactive environments, such as plasma-assisted combustion, is challenging, only a few experimental studies of the thermal impact of nanosecond plasma discharges have been done. Those were mainly limited to low pressure conditions (e.g., Zuzeek et al., 2011; Li et al., 2013; Lanier et al., 2014; Lefkowitz et al., 2015) or to nanosecond spark discharges with low repetition rates (e.g., Messina et al., 2007; Lo et al., 2014). To the best of our knowledge, the thermal impact of NRP glow discharges on a flame at atmospheric pressure has not yet been measured. This is the purpose of the present study, adopting conventional vibrational Coherent Anti-Stokes Raman Spectroscopy (CARS) as a thermometry technique. The evolution of the temperature across the flame front is presented and discussed.

\section{PLASMA-ASSISTED COMBUSTION SETUP}

The experimental setup used in this study has been previously described in Lacoste et al., (2017a). It comprises a jet burner, where an M-shape laminar methane-air premixed flame can be obtained at atmospheric pressure and room temperature (see Figure 1). The flame is stabilized on a central nozzle (quartz tube) of $7 \mathrm{~mm}$ inner diameter with a wall thickness of $1 \mathrm{~mm}$. At the center of the burner, a stainless steel rod of $0.85 \mathrm{~mm}$ diameter is inserted and used as a cathode for the NRP glow discharges. Simultaneously, the rod tip provides a flame anchoring point for 
the stabilization of the M-flame. A metallic ring, the anode, is located at the outlet of the quartz tube, surrounding it. The equivalence ratio and the nozzle exit mean velocity are fixed at 0.95 and $1.2 \mathrm{~m} / \mathrm{s}$, respectively. Note that the corresponding thermal power released by the flame is $113 \mathrm{~W}$. The NRP glow discharges are produced by high voltage pulses of $10 \mathrm{~ns}$ duration, $7 \mathrm{kV}$ amplitude, and a repetition frequency of $10 \mathrm{kHz}$ (FID, FPG 25-15 NM). A high voltage probe (Tektronix P6015A) and a current monitor (Pearson model 6585), were used to measure voltage and current. The conduction current is obtained after subtracting the capacitive displacement current from the total one. After synchronization, the voltage and conduction current have been multiplied and integrated, to obtain the energy deposition per plasma pulse. The average power of the plasma, determined from these measurements, is $1 \mathrm{~W}$, i.e, about $0.9 \%$ of the thermal power released by the flame. In order to obtain temperature profiles through the combustion and plasma region, the burner is installed on a 3-axis micrometric translation system.

Figures $1 \mathrm{~b}$ and $1 \mathrm{c}$ present direct visualizations of the flame enhanced by NRP discharges and without plasma, respectively. It is important to note that the NRP glow discharges follow the flame front (pink color originated from the $\mathrm{N}_{2}(\mathrm{C}-\mathrm{B})$ transition). The area where the plasma is the most luminous is just above the rod. Consequently, the most significant thermal impact of NRP discharges on the flame can be expected in this area.

The precise location of the combustion front, with and without enhancement by NRP glow discharges, has been determined from the $\mathrm{CH}(\mathrm{A}-\mathrm{X})$ chemiluminescence of the flame. An intensified charge couple device (ICCD) camera (PI-MAX, Princeton Instruments), equipped with a band pass filter centered at $430 \mathrm{~nm}$ (CVI Melles Griot F10-430.0-4-50.0M) is used. The exposure time is kept fixed at 98 s, and synchronized with the NRP discharges such as to collect the light only between plasma pulses. Thus, the chemiluminescence of $\mathrm{N}_{2}(\mathrm{C}-\mathrm{B})$ does not 
interfere with the emission of $\mathrm{CH}(\mathrm{A}-\mathrm{X})$. Figure 2 presents the $\mathrm{CH}(\mathrm{A}-\mathrm{X})$ emission of the flame alone (a), and of the flame enhanced by NRP glow discharges (b), after Abel transform. On the axis of the burner, the flame front is located $600+/-50 \mathrm{~m}$ above the rod. This distance is decreased to $500+/-50$ m by the application of NRP glow discharges.

The light emission from the discharges (mainly originated from the $\mathrm{N}_{2}(\mathrm{C}-\mathrm{B})$ transition) during the high-voltage pulses is presented in figure 2c. As it can also be observed in figure 1b, figure $2 \mathrm{c}$ shows that the highest density of plasma is located in between the top of the metallic rod and the flame front. As detailed in our recent work, Lacoste et al. (2017a), the strong effect of the plasma on the flame dynamics (with a change of about $5.5 \%$ of the flame surface, while the ratio of electrical power to the thermal power of the flame is less than $1 \%$ ) can be explained by a local increase in the reactivity of the fluid. In the gap between the rod and the flame, the NRP glow discharges change the gas properties and they locally increase the burning rate of the combustion front, leading to a change in the global surface of the flame. In the present study, it is investigated if this effect of the plasma can be attributed to a local heating of the gas.

\section{CARS SYSTEM}

Conventional broadband vibrational CARS of nitrogen is used to determine the temperature of the flame with and without enhancement by NRP glow discharges. Figure 3 presents a schematic of the CARS system. A frequency-doubled Nd:YAG laser delivers $650 \mathrm{~mJ}$ in about $10 \mathrm{~ns}$, with a repetition frequency of $10 \mathrm{~Hz}$ (Spectra-Physics, Quanta Ray). A part $(30 \%)$ of the second harmonic beam of the Nd:YAG $(532 \mathrm{~nm})$ pumps a modeless dye laser which emits the broadband Stokes beam, s, centered at $607 \mathrm{~nm}$ with a full width at half maximum of about $3 \mathrm{~nm}$. The dye solution is obtained by diluting Sulforhodamine 640 powder in methanol $(40 \mathrm{mg} / \mathrm{L})$. The rest of the second harmonic beam is split into two parallel beams to 
form the pump beams, $\mathrm{P} 1$ and $\mathrm{P} 2$, horizontally separated by $8 \mathrm{~mm}$ (BOXCARS phasematching configuration (Eckbreth, 1978)). The energy of each pump beam is $20 \mathrm{~mJ} / \mathrm{pulse}$, while it is $3 \mathrm{~mJ} /$ pulse for the Stokes laser. The three laser beams are focused at the same point using a $100 \mathrm{~mm}$ focal length converging lens. As the converging lens is not achromatic, a beam expander assembly is installed on the Stokes beam to ensure that all the beams focus at the same location. The probing volume, measured by translation of a glass sheet of 150 m thickness, is $600+/-50 \mathrm{~m}$ in length and $30+/-10 \mathrm{~m}$ in diameter.

The CARS signal, CARs, is collected through a converging lens (100 $\mathrm{mm}$ focal length), mirrors, and a band-pass filter (MaxDiode LD01-473/10-25, Semrock). A converging lens of 100 $\mathrm{mm}$ focal length focuses the CARS beam at the slit entrance of a spectrometer of $750 \mathrm{~mm}$ focal length, equipped with a grating of 1800 lines/mm (Acton SP2750, Princeton Instruments). An ICCD camera with $1024 \times 256$ pixels (PI-MAX 3, Princeton Instruments), is used as a detector. This choice has been dictated by the presence of plasma pulses emitting light in the range of wavelength of the CARS signal. Therefore, the intensifier has been set at its minimum (gain of 1 , to minimize the distortion of the spectra), and used as a fast shutter with an exposure time of 100 ns. The slit entrance is fixed at $100 \mathrm{~m}$, and the spectral resolution, which is measured as the full width at half maximum (FWHM), of the second harmonic of Nd:YAG laser line (at $532 \mathrm{~nm}$ ), is approximately $3 \mathrm{~cm}^{-1}$. A flow of argon at atmospheric pressure is used to determine the nonresonant CARS signal. In order to obtain an experimental spectrum, $I_{C A R S \text {, that }}$ can been compared with calculated spectra, the following post-processing of the data has been done:

$$
I_{C A R S}=\frac{\omega_{C A R S}-\omega_{\text {background }}}{\omega_{\text {NRCARS }}-\omega_{\text {NRbackground }}},
$$

where $\omega_{C A R S}$ is the measured spectrum to be analyzed, $\omega_{\text {background }}$ is the background spectrum obtained under the same experimental conditions than $\omega_{C A R S}$ but with a pump beam missing, 
$\omega_{N R C A R S}$ is the non-resonant CARS spectrum measured in argon, and $\omega_{\text {NRbackground }}$ is the background spectrum corresponding to the $\omega_{\text {NRCARS }}$ measurement.

The temperature profiles of the flame and the flame enhanced by NRP glow discharges are investigated by comparing $I_{C A R S}$ with spectra calculated with the Sandia CARSFT code (Palmer, 1989), which employs a least square error fit. An example of measured and calculated spectra is presented in figure 4. The red and blue spectra correspond to calculations with the lowest and highest values of the temperature, resulting in a reasonable fit of the hot band (from 2287 to $2300 \mathrm{~cm}^{-1}$ ). The average value of the envelop temperatures gives the gas temperature, while the uncertainty is obtained from their difference. In the example presented in figure 4 , the temperature of the gas is then $2028 \mathrm{~K}$ with an uncertainty of $+/-40 \mathrm{~K}$. The error bars in figures 5 , 6, and 7 have been determined by this method and therefore they correspond to the uncertainty in the fitting of the experimental spectra. They range from $+/-80 \mathrm{~K}$, down to $+/-40 \mathrm{~K}$ for the most precise data points. In order to increase the signal-to-noise ratio, experimental spectra are averaged over 500 measurements. A pulse/delay generator (Berkley Nucleonics, model 575-8C), synchronizes data acquisition and laser pulses with the NRP discharges.

\section{THERMAL CHARACTERIZATION}

Knowing the electrical energy per pulse, $E=100 \mu \mathrm{J}$, and the corresponding discharge volume, $V$, a thermodynamic estimation of the local heating of the gas by a plasma pulse could be obtained from:

$$
\Delta T=\frac{E}{\rho V C_{P}}
$$

where is the gas density and $C_{P}$ is the specific heat of the gas. However, as the discharges propagate in a complex environment, the determination of , $C p$, and $V$ is difficult. In addition, 
in air, depending on the experimental conditions, it has been shown that a large fraction of the energy deposition during NRP discharges can go into excitation, dissociation and ionization of the gas (e.g., Xu, 2014; Popov, 2001). This chemical activation of the gas would eventually lead to heating, but the time scale can be higher than a few hundreds of microseconds. Therefore, the fraction of energy that should be taken into account for the gas heating between plasma pulses could also be discussed.

Considering a limit case with the discharges propagating into fresh gases at $1000 \mathrm{~K}$ (i.e. in the preheat zone of the combustion), with a homogeneous energy deposition in the entire discharge volume (calculated as a cylinder of $0.6 \mathrm{~mm}$ diameter and $8 \mathrm{~mm}$ long), and with only $25 \%$ of the energy going into gas heating, the increase of gas temperature should be about $14 \mathrm{~K}$. The other limit case corresponds to an electrical energy deposition concentrated above the rod (in the area of maximum light emission from the discharges, i.e. up to $2 \mathrm{~mm}$ far from the rod), with $100 \%$ of the energy going into gas heating, upstream of the combustion front. The increase of gas temperature should then be about $480 \mathrm{~K}$, leading to a local increase of the adiabatic temperature of combustion of $230 \mathrm{~K}$. If $14 \mathrm{~K}$ can be considered as negligible, a local increase of the fresh gases temperature of $480 \mathrm{~K}$ may have dramatic effects on the combustion. Therefore, a more precise determination of the thermal impact of NRP glow discharges is necessary.

Figure 5 presents the gradient of temperature above the central rod of the burner, without enhancement by NRP glow discharges. At $100 \mathrm{~m}$ above the rod, the temperature is $1030 \mathrm{~K}$. It increases up to $2110 \mathrm{~K}$, at $\mathrm{z}=1.5 \mathrm{~mm}$. This is in agreement with the location of the peak of $\mathrm{CH}(\mathrm{A}-\mathrm{X})$ emission, located at $\mathrm{z}=0.6 \mathrm{~mm}$ (see figure 2). For these experimental conditions, the adiabatic flame temperature, $\mathrm{T}_{\mathrm{ad}}$, is $2185 \mathrm{~K}$. For $\mathrm{z}$ greater than $2 \mathrm{~mm}$, taking into account the small diameter of the rod $(0.85 \mathrm{~mm})$, the cooling down of the gases by heat losses to the rod can 
be neglected. However, the measured temperature is about $4 \%$ lower than $\mathrm{T}_{\mathrm{ad}}$. This difference can be explained by a combination of uncertainty in the temperature measurements and uncertainty in the equivalence ratio. Indeed, the accuracy of the mass flow controllers used for this study is $1 \%$, inducing about $1 \%$ uncertainty of the adiabatic flame temperature.

To have a chance to capture the maximal thermal impact of NRP discharges on the flame, the temporal evolution of the temperature on the axis of the burner at $\mathrm{z}=1 \mathrm{~mm}$ is investigated. This height is chosen as a compromise between the location of NRP discharges on the axis of the burner, i.e., up to $500 \mathrm{~m}$ above the rod (see figures $1 \mathrm{~b}$ and 2 ), and a zone where the temperature should be uniform within the probing area. Indeed, the diameter of NRP discharges, where heating of the gas may be induced in a non-uniform manner, is about $500 \mathrm{~m}$ (see Stancu et al., 2010). At z = $1 \mathrm{~mm}$, without plasma enhancement, the temperature is $2000+/-40 \mathrm{~K}$.

Figures 6 presents the evolution of the temperature between plasma pulses when NRP glow discharges are applied for more than one minute, i.e., at the steady state regime of plasma actuation. From $500 \mathrm{~ns}$ to $99 \mathrm{~s}$ after a plasma pulse, the temperature is constant, within the uncertainty of the measurements. In average, the temperature measured during NRP glow discharges actuation is $2024 \mathrm{~K}$, i.e., within the measurement uncertainty of the temperature measured without plasma. Therefore, it can be reasonably concluded that, for this configuration, the thermal impact of NRP glow discharges is in the uncertainty of the technique, i.e., $+/-40 \mathrm{~K}$ for this range of temperatures. Compared to an increase of the burnt gases temperature of $230 \mathrm{~K}$, as it can be expected for a purely thermal effect of electrical discharges, the thermal impact of NRP glow discharges is negligible. Therefore, the chemical activation of the gas by NRP glow discharges seems to be a realistic explanation of the plasma action on the combustion. 
Figure 7 compares the temperature radial profiles, at $\mathrm{z}=1$, obtained with and without enhancement by NRP glow discharges. The temperatures were measured 5 s after a highvoltage pulse. Only the central part of the flame (between 0.75 and $1.75 \mathrm{~mm}$ ) seems to be affected by the plasma. With plasma enhancement, the hot zone over the rod is broader than for the flame alone. This can be explained by the anchoring effect of the NRP discharges to the flame, as already observed by Lacoste et al., (2013a). This effect of NRP glow discharges on the temperature profile is in agreement with its effect on the shape of the flame, as can be observed on figure 2. It could be attributed to an increase in the local burning velocity due to chemical activation of the fluid by the plasma. Besides, we may conceive that the radicals generated through the NRP glow discharges can inhibit a wall quenching which results in a standoff distance as a dark space between a nozzle and the base of a nozzle attached flame.

\section{CONCLUSION}

The thermal impact of NRP glow discharges on a lean methane-air laminar flame is investigated by CARS measurements. Using an M-shape laminar methane-air premixed flame, it is shown that the shape of the flame can be modified with a small plasma power, which is mere $0.9 \%$ of the thermal power released by the flame. This change in the flame shape, as well as the strong effect of NRP glow discharges on the flame dynamics, presented in Lacoste et al., 2017a, cannot be explained by an increase of the flame temperature. Indeed, CARS measurements show that the increase in temperature due to NRP glow discharges actuation is in the uncertainty of the technique, i.e., $+/-40 \mathrm{~K}$ for this range of temperatures. Consequently, the chemical activation of the gas by NRP glow discharges seems to be a realistic explanation of the plasma effect. 
In order to corroborate this result, our future work will focus on temperature measurement by CARS during NRP glow discharges generated in a combustion zone. For NRP discharges in air, the vibrational temperature of nitrogen is usually different than the gas temperature, therefore vibrational CARS cannot be used as a thermometry technique for determining the heating of the gas. In another hand, at atmospheric pressure, the rotational temperature of nitrogen can be used to determine the gas temperature (see e.g., Rusterholtz, 2013). Thus, rotational CARS of nitrogen may be an adequate technique to determine the local gas heating by NRP glow discharges in combustion environment.

\section{AKNOWLEDGMENT}

The research reported in this publication was supported by Center Competitive Funding from King Abdullah University of Science and Technology (KAUST).

\section{REFERENCES}

Do, H., Im, S.K., Cappelli, M.A., and Mungal, M.G. 2010. Plasma assisted flame ignition of supersonic flows over a flat wall. Combust. Flame, 157, 2298.

Eckbreth, A.C. 1978. BOXCARS: Crossed-beam phase-matched CARS generation in gases. Appl. Phys. Lett., 32, 421.

Ju, Y., and Sun, W. 2015. Plasma assisted combustion: dynamics and chemistry. Progress Energy Combust. Sci., 48, 21.

Lacoste, D.A., Moeck, J.P., Durox, D., Laux, C.O., and Schuller, T. 2013b. Effect of nanosecond repetitively pulsed discharges on the dynamics of a swirl-stabilized lean premixed flame. $J$. Engineer. Gas Turbines Power, 135, 101501. 
Lacoste, D.A., Moeck, J.P., Paschereit, C.O., and Laux, C.O. 2013c. Effect of plasma discharges on nitric oxide emissions in a premixed flame. J. Propulsion Power, 29(3), 748.

Lacoste, D.A., Moeck, J.P., Roberts, W.L., Chung, S.H., and Cha, M.S. 2017a. Analysis of the step responses of laminar premixed flames to forcing by non-thermal plasma. Proc. Combust. Inst., 36, 4145-4153.

Lacoste, D.A., Xiong, Y., Moeck, J.P., Chung, S.H., Roberts, W.L., and Cha, M.S. 2017 b. Transfer functions of laminar premixed flames subjected to forcing by acoustic waves, AC electric fields, and non-thermal plasma discharges. Proc. Combust. Inst., 36, 4183-4192.

Lacoste, D.A., Xu, D.A., Moeck, J.P., and Laux, C.O. 2013a. Dynamic response of a weakly turbulent lean-premixed flame to nanosecond repetitively pulsed discharges. Proc. Combust. Inst., 34, 3259.

Lanier, S., Bowman, S., Burnette, D., Adamovich, I.V., and Lempert, W.R. 2014. Time-resolved temperature and $\mathrm{O}$ atom measurements in nanosecond pulse discharges in combustible mixtures. J. Phys. D: Appl. Phys., 47, 445204.

Lefkowitz, J.K., Guo, P., Rousso, A., and Ju, Y.G. 2015. Species and temperature measurements of methane oxidation in a nanosecond repetitively pulsed discharge. Philo. Trans. Royal Society A: Math. Phys. Engineer. Sci., 373(2048), 20140333.

Li, T., Adamovich, I.V., and Sutton, J.A. 2013. A burner platform for examining the effects of non-equilibrium plasmas on oxidation and combustion chemistry. Combust. Sci. Technol., $185,990$.

Lo, A. Cessou, A., Boubert, P., and Vervisch, P. 2014. Space and time analysis of the nanosecond scale discharges in atmospheric pressure air: I. Gas temperature and vibrational distribution function of $\mathrm{N}_{2}$ and $\mathrm{O}_{2}$. J. Phys. D: Appl. Phys., 47, 115201. 
Messina, D., Attal-Trétout, B., and Grisch, F. 2007. Study of a non-equilibrium pulsed nanosecond discharge at atmospheric pressure using anti-Stokes Raman scattering. Proc. Combust. Inst., 31, 825.

Palmer, R.E. 1989. The CARSFT computer code for calculating coherent anti-Stokes Raman spectra: user and programmer information. SANDIA National Laboratory, Report SAND898206

Pai, D.Z., Lacoste, D.A., and Laux, C.O. 2010. Transitions between corona, glow, and spark regimes of nanosecond repetitively pulsed discharges in air at atmospheric pressure. J. Appl. Phys., 107, 093303.

Pai, D.Z., Stancu, G.D., Lacoste, D.A., and Laux, C.O. 2009. Nanosecond repetitively pulsed discharges in air at atmospheric pressure - the glow regime. Plasma Sources Sci. Technol., 18, 045030 .

Pilla, G., Galley, D., Lacoste, D.A., Lacas, F., Veynante, D., and Laux, C.O. 2006. Stabilization of a turbulent premixed flame using a nanosecond repetitively pulsed plasma. IEEE Trans. Plasma Sci., 34(6), 2471.

Pilla, G., Lacoste, D.A., Veynante, D., and Laux, C.O. 2008. Stabilization of a swirled propaneair flame using a nanosecond repetitively pulsed plasma. IEEE Trans. Plasma Sci., 36(4), 940.

Popov, N.A. 2001. Investigation of the mechanism for rapid heating of nitrogen and air in gas discharges. Plasma Phys. Rep., 27, 886-96.

Rusterholtz, D.L., Lacoste, D.A., Stancu, G.D., Pai, D.Z., and Laux, C.O. 2013. Ultrafast heating and oxygen dissociation in atmospheric pressure air by nanosecond repetitively pulsed discharges. J. Phys. D: Appl. Phys., 46, 464010. 
Stancu, G.D., Kaddouri, F., Lacoste, D.A., and Laux, C.O. 2010. Atmospheric pressure plasma diagnostics by OES, CRDS and TALIF. J. Phys. D: Appl. Phys., 43, 124002.

Starikovskaia S.M. 2014. Plasma-assisted ignition and combustion: nanosecond discharges and development of kinetic mechanisms. J. Phys. D.: Appl. Phys., 47(35), 353001.

Starikovskiy, A., and Aleksandrov, N. 2013. Plasma-assisted ignition and combustion. Progress Energy Combust. Sci., 39, 61.

Tholin, F., Lacoste, D.A., and Bourdon, A. 2014. Influence of fast-heating processes and O atom production by a nanosecond spark discharge on the ignition of a lean $\mathrm{H}_{2}$-air premixed flame. Combust. Flame, 161(5), 1235.

Xu, D.A., Shneider, M.N., Lacoste, D.A., and Laux, C.O. 2014. Thermal and hydrodynamic effects of nanosecond discharges in atmospheric pressure air. J. Phys. D: Appl. Phys., 47, 235202.

Yin, Z., Takashima, K., and Adamovich, I.V. 2011. Ignition time measurements in repetitive nanosecond pulse hydrogen-air plasmas at elevated initial temperatures. IEEE Trans. Plasma Sci., 39(12), 3269.

Zuzeek, Y., Bowman, S., Choi, I., Adamovich, I.V., and Lempert, W.R. 2011. Pure rotational CARS studies of thermal energy release and ignition in nanosecond repetitively pulsed hydrogen-air plasmas. Proc. Combust. Inst., 33, 3225.

Figure 1 (a) Schematic of the experimental setup, and photographs of the methane-air M-flame, equivalence ratio 0.95 , thermal power $113 \mathrm{~W}$, with (b) and without (c) NRP glow discharges 
at $10 \mathrm{kHz}$.
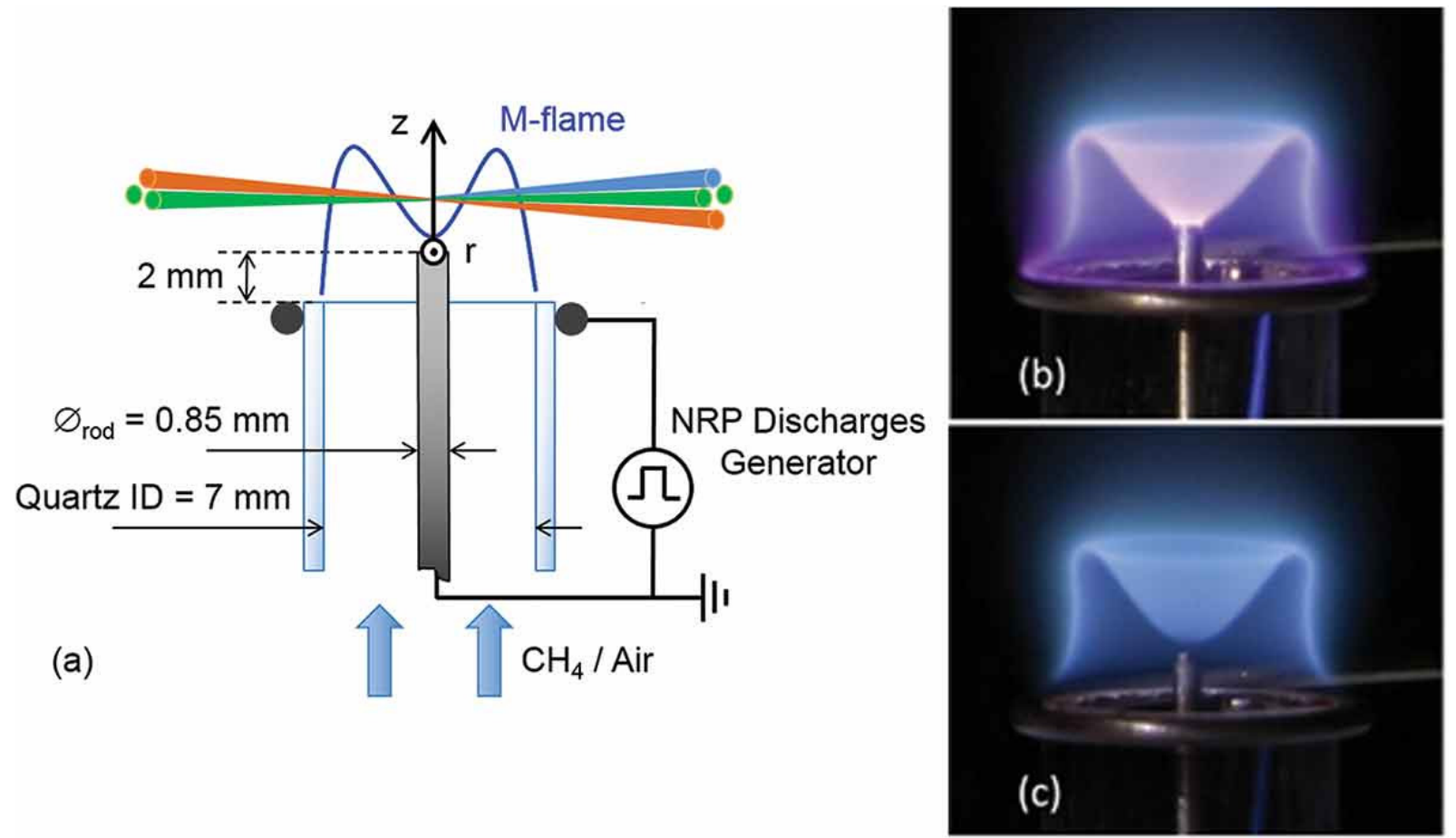
Figure 2 Abel transformed images of (a) $\mathrm{CH}(\mathrm{A}-\mathrm{X})$ chemiluminescence of the flame, (b) $\mathrm{CH}(\mathrm{A}$ X) chemiluminescence of the flame enhanced by NRP glow discharges at $10 \mathrm{kHz}$, and (c) N2(C-B) emission of the NRP glow discharges. The exposure time is fixed at 98 ?s for $\mathrm{CH}(\mathrm{A}-\mathrm{X})$ emission, and 1 ?s for N2(C-B) emission, and synchronized with the plasma pulses, to avoid parasitic light.

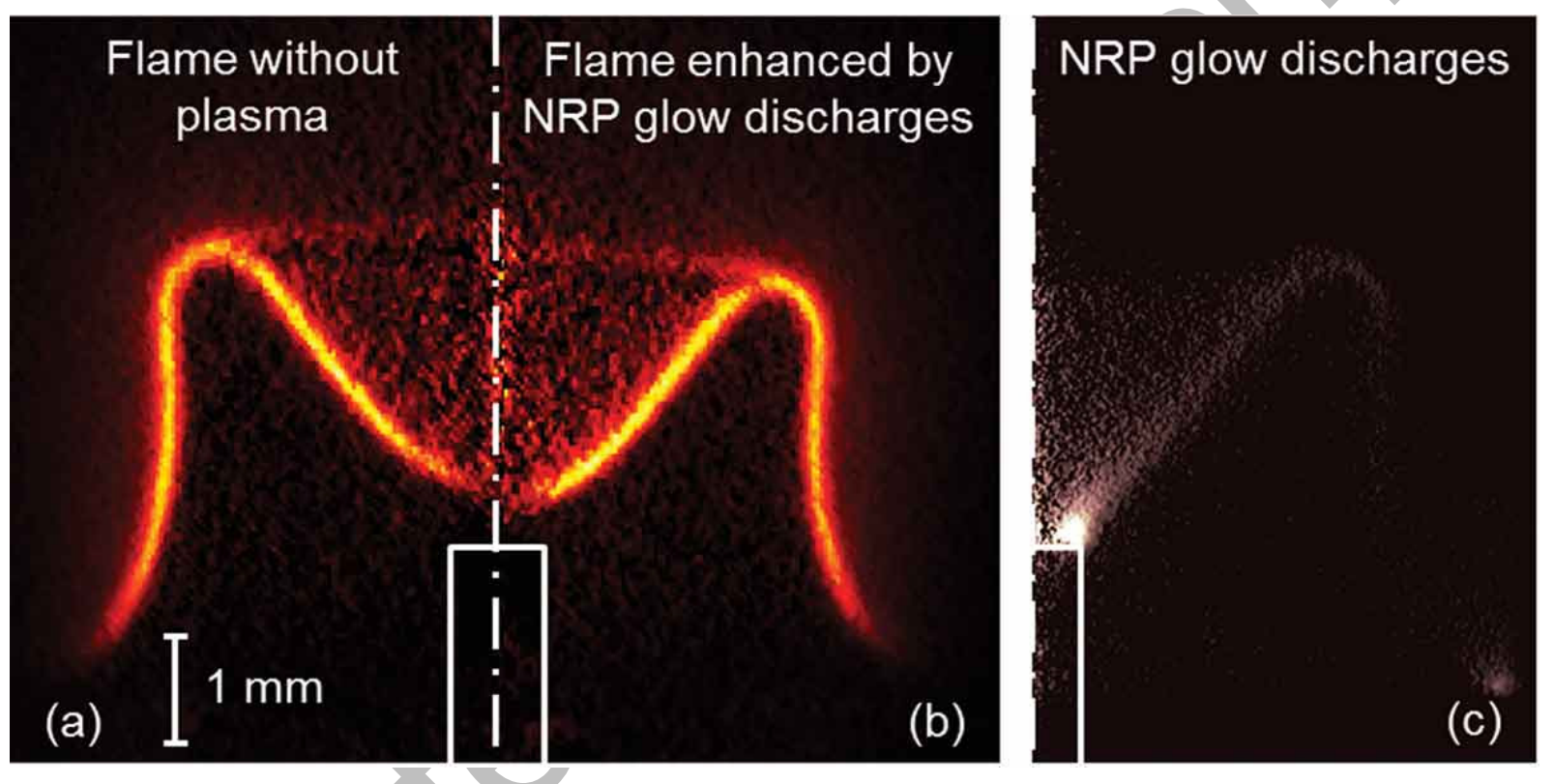


Figure 3 Schematic of the conventional broadband CARS system used for thermometry of the flame.

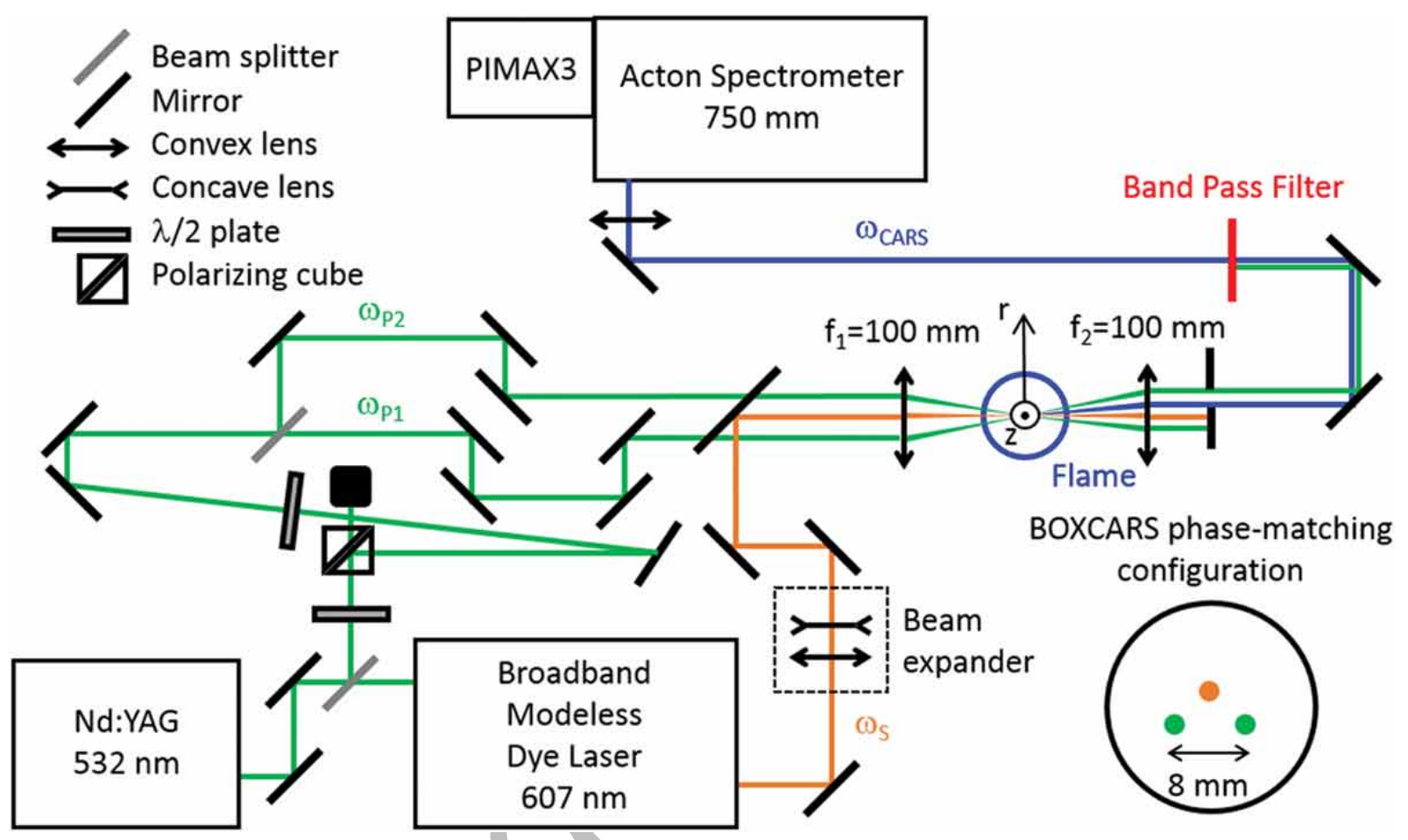


Figure 4: Example of the square root of the experimental spectrum, ICARS, measured $1 \mathrm{~mm}$ above the rod, for a flame enhanced by NRP glow discharges, 50 ?s after a plasma pulse (black), and spectra calculated with the CARSFT code (Palmer, 1989), for temperatures of $1988 \mathrm{~K}$ (red) and $2068 \mathrm{~K}$ (blue).

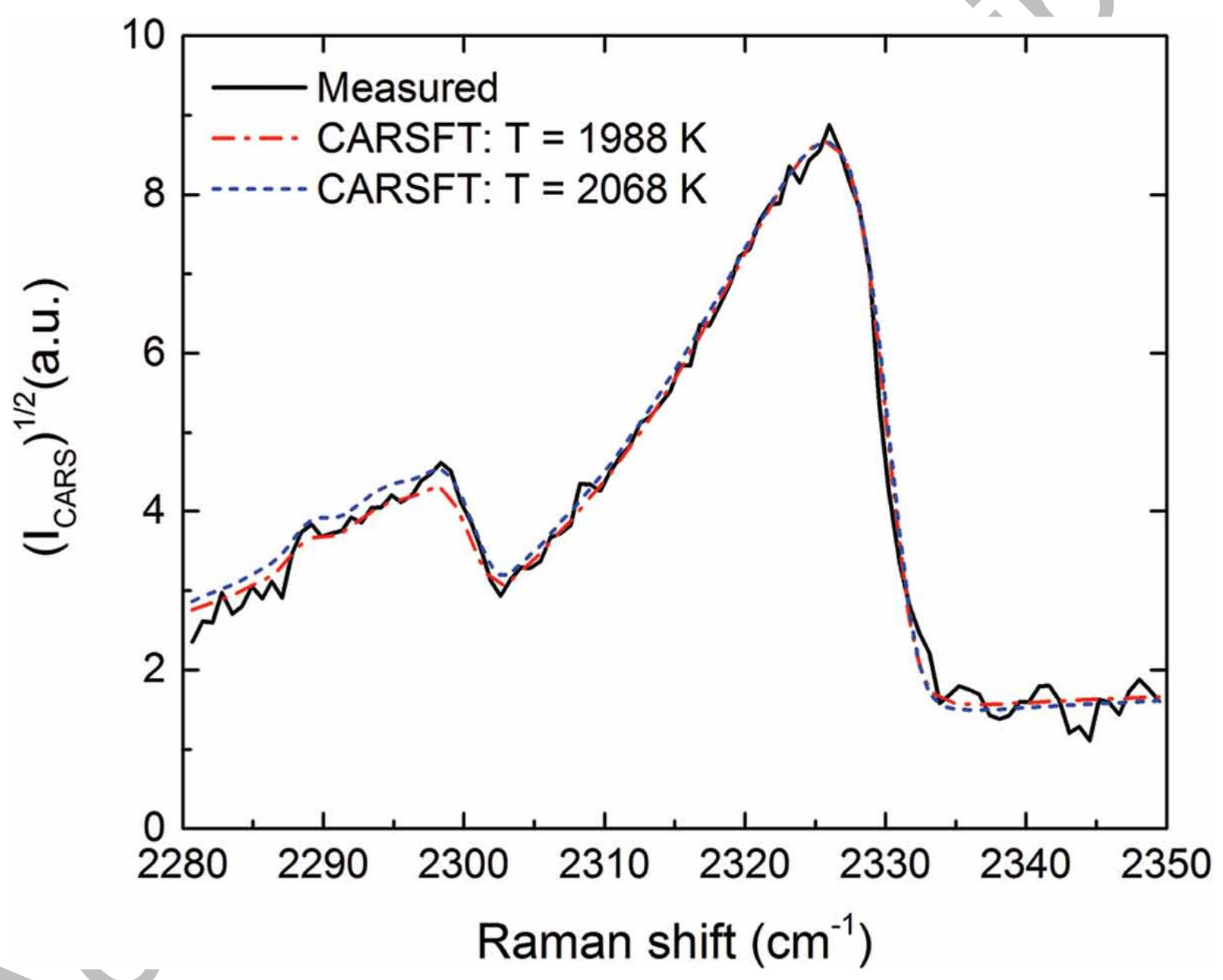


Figure 5 Temperature gradient above the rod for the flame without enhancement by NRP discharges.

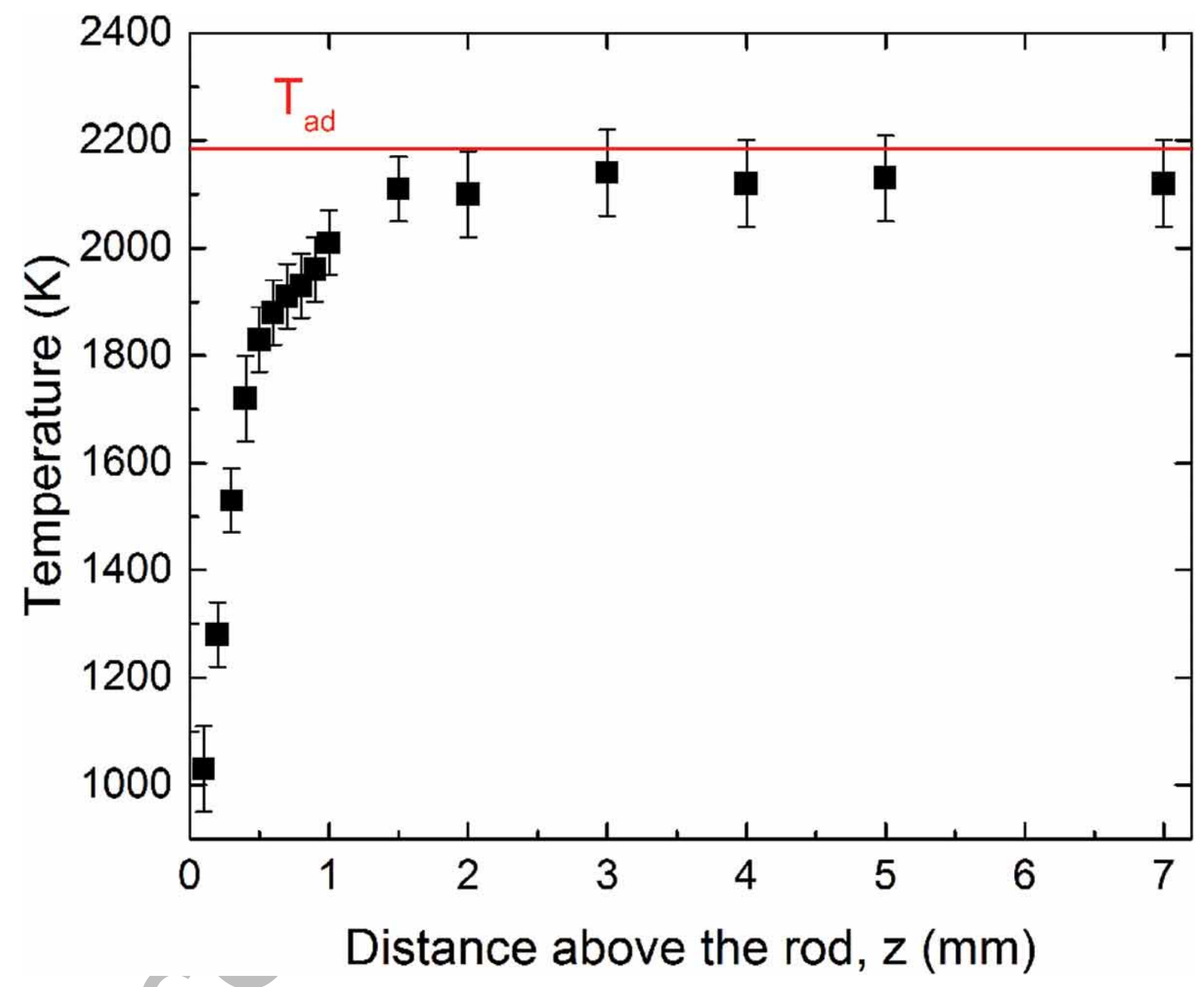


Figure 6: Temporal evolution of the temperature measured on the axis of the burner, at $\mathrm{z}=1 \mathrm{~mm}$, between plasma pulses produced by NRP glow discharges at $10 \mathrm{kHz}$. The corresponding temperature for a flame, without plasma enhancement, is $2000+/-40 \mathrm{~K}$.

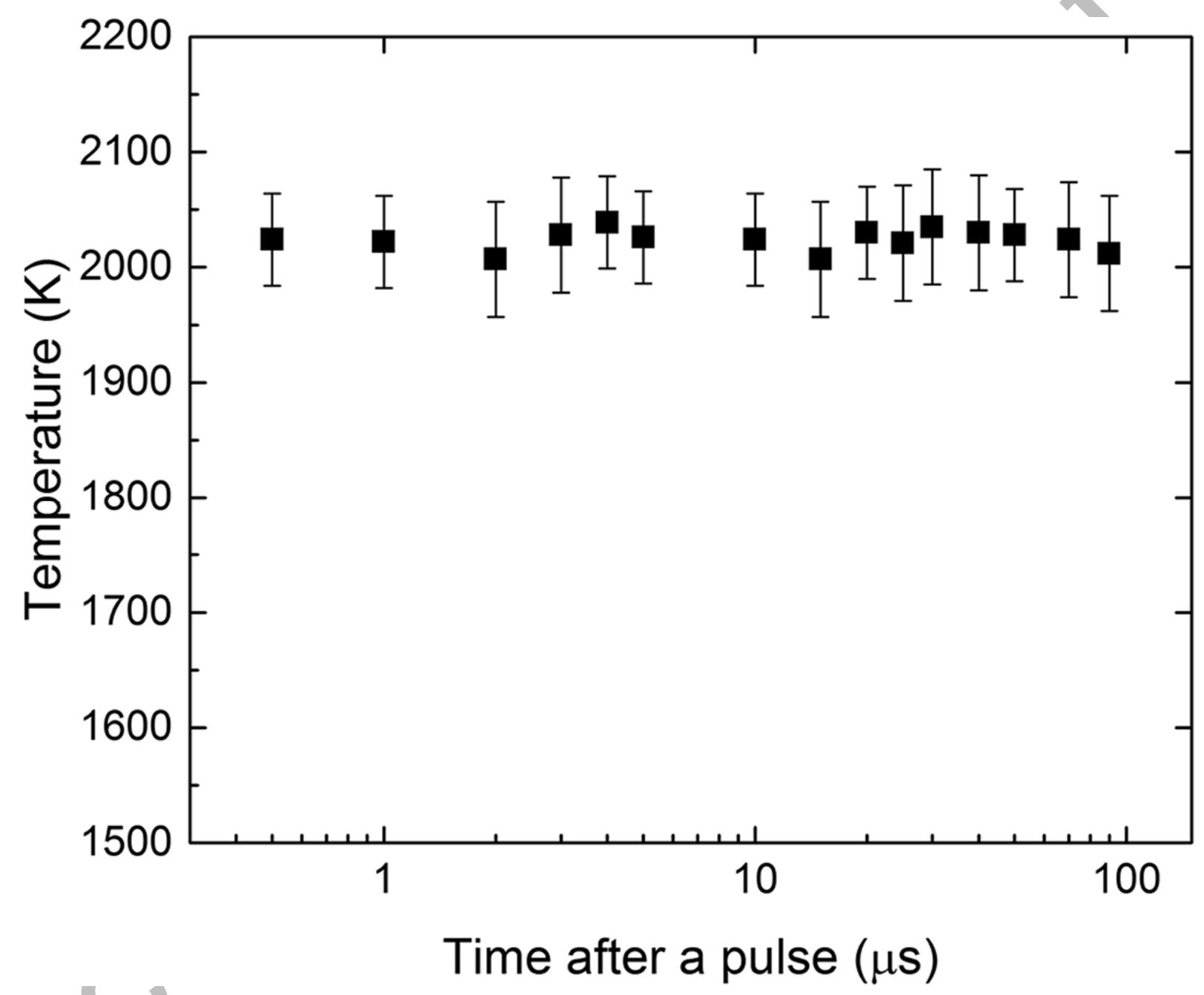


Figure 7 Temperature profile measured at $\mathrm{z}=1 \mathrm{~mm}$, for a flame enhanced by NRP glow discharges, 5 ?s after a plasma pulse (red), and for a flame without plasma enhancement (black).

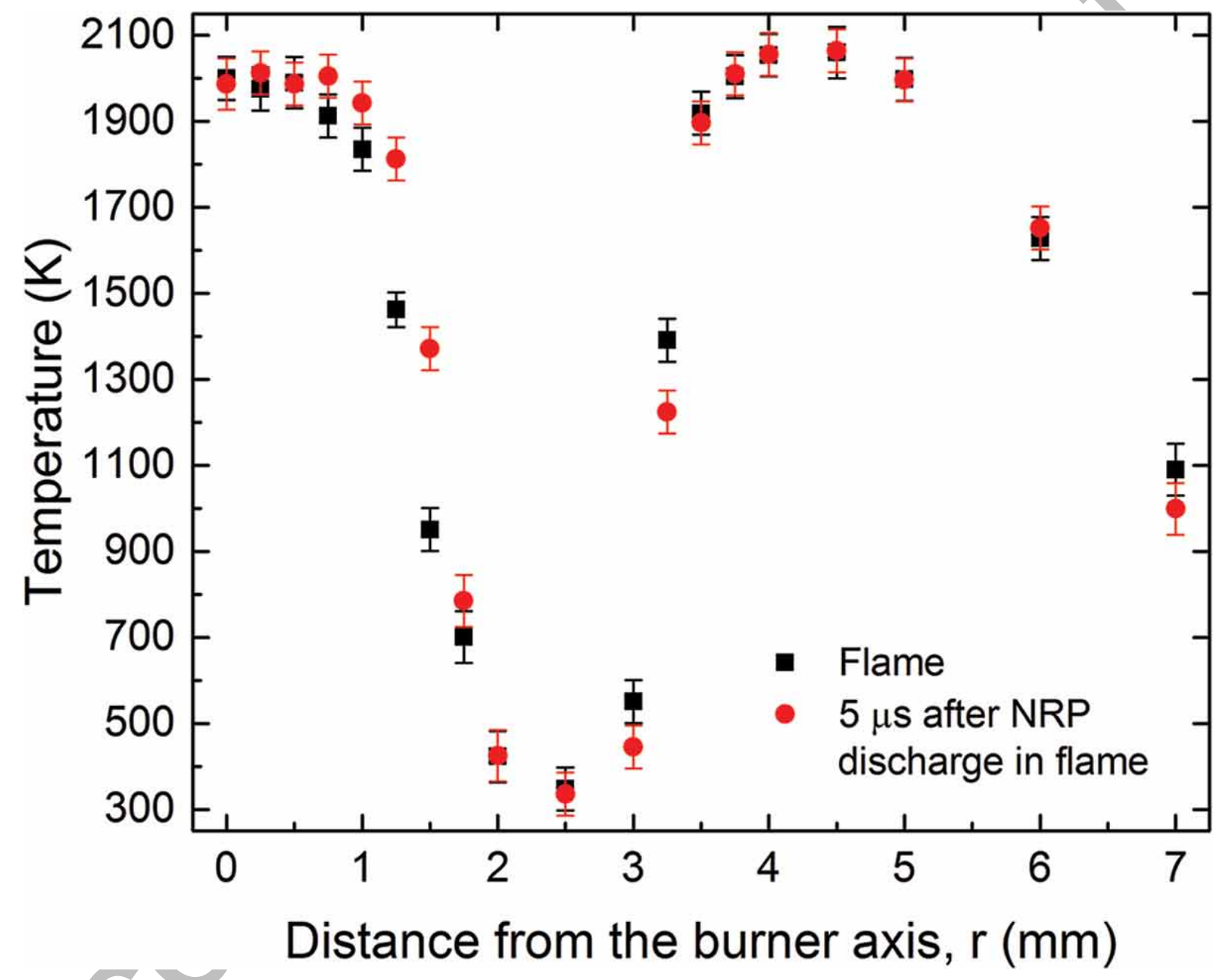

